10. J. R. Teague, R. Gerson, W. J. James, Solid State Commun. 8, 1073 (1970)

11. Yu. E. Roginskaya, Yu. Ya. Tomashpol'skii, Yu. N Venevtsev, V. M. Petrov, G. S. Zhdanov, Sov. Phys. JETP 23, 47 (1966).

12. S. V. Kiselev, R. P. Ozerov, G. S. Zhdanov, Sov. Phys. Dokl. 7, 742 (1963).

13. F. Kubel, H. Schmid, Acta Crystallogr. B 46, 698 (1990).

14. K. Ueda, H. Tabata, T. Kawai, App. Phys. Lett. 75, 555 (1999).

15. C. B. Eom, R. J. Cava, R. M. Fleming, J. M. Phillips, Science 258, 1766 (1992).

16. M. J. Haun, E. Furman, S. J. Jang, L. E. Cross, Ferroelectrics 99, 63 (1989).

17. N. A. Pertsev, A. G. Zembilgotov, A. K. Tagantsev, Phys. Rev. Lett. 80, 1988 (1998)

18. G. Kresse, J. Hafner, Phys. Rev. B 47, 558 (1993).
19. _ Phys. Rev. B 54, 11169 (1996).

20. G. Kresse, J. Joubert, Phys. Rev. B 59, 1758 (1999).

21. The ABINIT code is a common project of the Université Catholique de Louvain, Corning Incorporated, and other contributors (www.abinit.org). See X. Gonze et al., Comput. Mater. Sci. 25, 478 (2002).

22. W. Zhong, R. D. King-Smith, D. Vanderbilt, Phys. Rev. Lett. 72, 3618 (1994).

23. R. D. King-Smith, D. Vanderbilt, Phys. Rev. B 47, 1651 (1993).

24. D. Vanderbilt, R. D. King-Smith, Phys. Rev. B 48, 4442 (1993).

25. M. Mahesh Kumar, S. Srinath, G. S. Kumar, S. B. Suryanarayana, J. Magn. Magn. Mater. 188, 203 (1998).

26. H. G. Rajaram, thesis, Pennsylvania State University (1991).

27. J. Wang et al., data not shown.
28. We thank L. V. Saraf and V. Kulkarni for help with $x$-ray diffraction and Rutherford Backscattering measurements. J.B.N. and K.M.R thank D. Vanderbilt for useful discussions. Supported by the Office of Naval Research (grant MURI N000140110761) and the NSF (grant MRSEC DMR-00-80008. It also benefits from the support of the NSF grant DMR0095166

\section{Supporting Online Material}

www.sciencemag.org/cgi/content/full/299/5613/1719/

DC1

SOM Text

Figs. S1 and S2

References and Notes

19 November 2002; accepted 3 February 2003

\title{
Taming Winfree Turbulence of Scroll Waves in Excitable Media
}

\author{
Sergio Alonso, ${ }^{1}$ Francesc Sagués, ${ }^{1}$ Alexander S. Mikhailov ${ }^{2 *}$
}

\begin{abstract}
Winfree turbulence of scroll waves is a special kind of spatiotemporal chaos that exists exclusively in three-dimensional excitable media and is currently considered one of the principal mechanisms of cardiac fibrillation. A chaotic wave pattern develops through the negative-tension instability of vortex filaments, which tend to spontaneously stretch, bend, loop, and produce an expanding tangle that fills up the volume. We demonstrate that such turbulence can readily be controlled by weak nonresonant modulation of the medium excitability. Depending on the forcing frequency, either suppression or induction of turbulence can be achieved.
\end{abstract}

About 10 years ago, Winfree suggested that three-dimensional (3D) excitable media may possess a special mechanism of wave turbulence, different in principle from the breakup of spiral waves in two dimensions. Spatiotemporal chaos emerges via the disorderly dynamics shown by the vortex filaments of scroll waves, which "snake about, fragment, and close in rings" (1). In his view, this process could provide an explanation for the development of fibrillation and the occurrence of sudden cardiac death in healthy people. In subsequent discussion, his analysis of cardiological data was criticized (2) and it was also pointed out that sometimes breakup of waves occurs more easily in large volumes than in thin slabs (3). Nonetheless, the turbulence described by Winfree has indeed been found in general models of excitable media. Its origin lies in the effect of negative tension of vortex filaments (4-7). Such filaments, which represent the cores of rotating scroll waves, tend to stretch themselves and to form loops and complex tangles. This behavior is characteristic for media with relatively low excitability, where the rotation period of

1Departament de Química Física, Universitat de Barcelona, Avenida Diagonal 647, 08028 Barcelona, Spain. ${ }^{2}$ Abteilung Physikalische Chemie, Fritz-HaberInstitut der Max-Planck-Gesellschaft, Faradayweg 4-6, 14195 Berlin, Germany.

*To whom correspondence should be addressed. Email: mikhailov@fhi-berlin.mpg.de spiral waves is large and the medium completely recovers after each propagating wave. In recent realistic simulations of cardiac arrhythmias, this form of fibrillation has been seen under ischemic conditions, in cardiac tissue deprived of oxygen (8). Scroll waves are also possible in other excitable media, such as the chemical Belousov-Zhabotinsky reaction (9) or the slime mold Dictyostelium discoideum (10). Experiments on taming excitation waves (11-13), oscillatory patterns (14-15), and chemical turbulence (16) in thin aqueous layers and surface chemical reactions have recently attracted much attention. In excitable 3D media, only the effects of spatial gradients $(17-18)$, resonant periodic forcing (19), and noise (20) on scroll waves have previously been discussed. Here, we show that the Winfree turbulence of scroll waves is amenable to control by uniform periodic forcing. Both suppression and induction of such turbulence, depending on the forcing period, are demonstrated.

In its transverse cross section, a scroll wave looks like a spiral. Such spirals are stacked one upon another to form a scroll-shaped pattern. The scroll rotates around a central filament characterized by zero excitation amplitude. This filament can be straight or curved; it may also form loops and close into contracting or expanding rings. Our analysis is based on the general Barkley model (21), whose parameter space is shown in Fig. 1. Inside a broad region, characterized by decreased excitability, expanding scroll rings that give rise to Winfree turbulence were observed. An example of turbulence development is presented in Fig. 2, A to E, where thick yellow lines show the filaments around which scroll waves are rotating. The inhibitor
Fig. 1. Parameter space of the Barkley model. Evolution of the activator $u$ and inhibitor $v$ variables is governed in this model by two equations: $\partial u / \partial t=\varepsilon^{-1} u(1-u)[u-(v+$ $b) / a)]+\nabla^{2} u$ and $\partial v / \partial t=u-v_{1}$ where $\varepsilon$ is the ratio of the characteristic activator and inhibitor time scales, $\varepsilon$ is fixed at 0.02 here and a and $b$ are parameters of the model, $b$ determining the excitation threshold. Stable 2D spiral waves are found in numerical simulations inside the entire white region, and meandering of spiral waves takes place in region MS. The $2 \mathrm{D}$ medium does not support excitation waves in region NW, is subexcitable (wave fragments shrink) in region $\mathrm{SE}$, and is bistable in region $\mathrm{BI}$. A line divides the white region into the domains where collapsing (CS) and expanding (ES) scroll rings are observed. This boundary was determined by numerical 3D simulations that assume axial symmetry of the rings. The symbols (open and solid dots and squares) indicate the points at which numerical simulations were performed. NW, no waves; SE, subexcitable; MS, meandering spirals; Bl, bistable. 

waves are shown on the back and bottom planes of the cube-shaped medium. First, the expanding filament is circular (Fig. 2A). As it grows, transverse deformations develop (Fig. 2B). Soon, the length of the filament rapidly increases; it loops out and forms an irregular expanding tangle (Fig. 2, C and D). The tangle consists of only one connected filament (no closed rings are budding up) until it touches a boundary plane. After that, the filament gets fragmented into many pieces that span the medium. We have checked that the resulting irregular wave pattern is characterized by rapid decay of spatial autocorrelation functions and by a broad frequency spectrum.

Periodic time-dependent forcing was introduced into the model by periodic variation of the excitation threshold. Such forcing has been switched on in Fig. 2E, and its action is illustrated in Fig. 2, F to I. The application of periodic forcing led to suppression of turbulence. Curved vortex filaments shrank and disappeared. An additional effect of external forcing was that the crossover of filaments, leading to the generation of small, separate, closed rings, became possible (22). Such small distributions corresponding to these excitation

rings also rapidly shrank and disappeared.

A series of simulations revealed that the effect of forcing depends on the relation between the forcing period $T_{\mathrm{f}}$ and the rotation period $T_{0}$ of spiral waves. When $T_{\mathrm{f}}<T_{0}$, sufficiently strong forcing eliminated turbulence and damped excitation. Sometimes, a stable straight scroll wave with a filament connecting opposite boundary planes was seen at the end. However, when the forcing period exceeded the rotation period of the scroll waves $\left(T_{\mathrm{f}}>T_{0}\right)$, suppression of turbulence was not observed.

To analyze the mechanism of turbulence suppression, we investigated the action of periodic forcing on relatively small scroll rings (which were substantially larger than their filament thickness, but still small when compared with the dimensions of the medium). First, model parameters were chosen from region ES in Fig. 1 and weak forcing was applied. Rapid forcing $\left(T_{\mathrm{f}}<T_{0}\right)$ of expanding scroll rings stopped their expansion and led to the shrinking and disappearance of scroll rings, accompanied by some oscillation of their radius (Fig. 3A). Slow forcing with $T_{\mathrm{f}}>T_{0}$ had an opposite effect and enhanced the expansion.
A

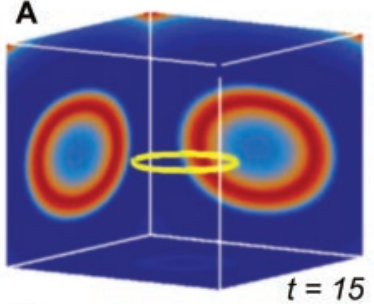

D

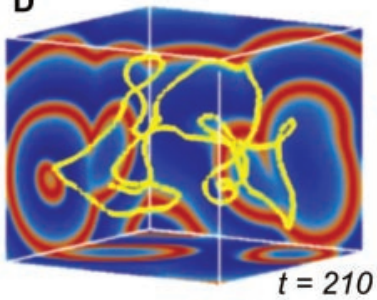

G

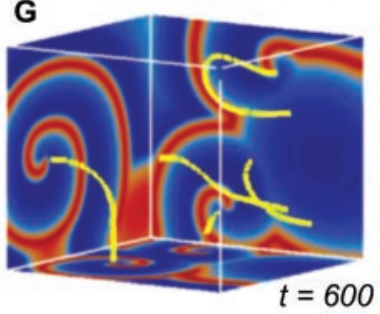

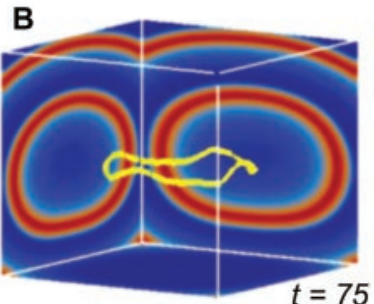
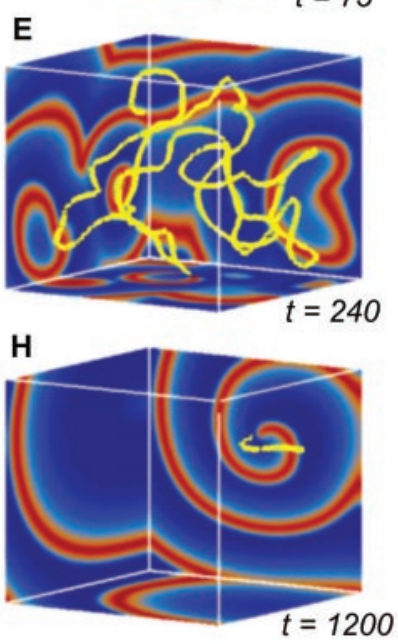

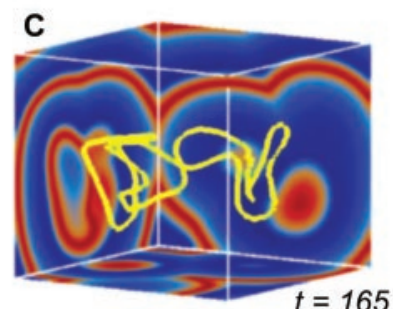

F
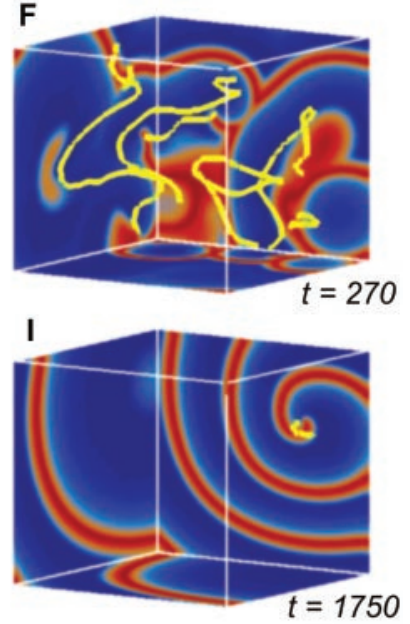

Fig. 2. Winfree turbulence of vortex filaments and its suppression by external forcing. (A to E) Development of turbulence. Periodic forcing $b(t)=b_{0}+b_{f} \cos \left(\omega_{\mathrm{f}} t\right)$ with amplitude $b_{\mathrm{f}}=0.03$ and frequency $\omega_{\mathrm{f}}=1.2$ is introduced at $t=240$. ( $\mathrm{F}$ to $\mathrm{I}$ ) Suppression of turbulence by the periodic forcing. In this example, suppression of turbulence took about 300 of the scroll waves' rotation periods. Yellow lines show filaments of rotating scroll waves. Inhibitor distributions on three back planes are displayed, with red corresponding to high concentrations and blue corresponding to low concentrations. Numerical simulations are given for the Barkley model with $a=1.1, b_{0}=0.19$, and $\varepsilon=$ 0.02 in a cube of linear size $L_{0}=60$ with no-flux boundary conditions. The time moments are indicated below each panel. The initial scroll ring was produced by creating a spherical wave and then resetting half of the medium to the equilibrium steady state. The rotation frequency of spiral waves for this set of parameters was $\omega_{0}=1.19$.
Periodic forcing of collapsing scroll rings in parameter region CS in Fig. 1 was also investigated. The application of rapid forcing with $T_{\mathrm{f}}<T_{0}$ increased the rate of collapse. In contrast, forcing with $T_{\mathrm{f}}>T_{0}$ slowed down the collapse. If such forcing was sufficiently strong, it stopped the collapse and induced the expansion of scroll rings, accompanied by periodic oscillations of their radius (Fig. 3B). Subsequently, such forced scroll rings underwent instabilities that led to stretching of their filaments, formation of loops, and development of turbulence. This turbulence was induced by the external forcing and was maintained as long as forcing was present. When forcing was terminated, the filaments underwent collapse and disappeared. A special feature of the stimulationinduced turbulence was that, in contrast to the free turbulence of scroll waves displayed in Fig. 2 , spontaneous budding of small scroll rings from larger filaments was observed.

Generally, the evolution of scroll rings is governed (23) by the rate law $\dot{R}=-\alpha / R$, where $R$ is the radius of the filament and $\alpha$ is the filament tension coefficient. The validity of this equation for both contracting and expanding rings has been numerically verified (4). An analytical expression for the tension coefficient $\alpha$ was obtained through the phenomenological kinematic theory of scroll waves $(5,24)$. Within the framework of kinematics, the linear response of scroll rings on resonant periodic forcing has been previously investigated (25). We extended (26) this kinematic perturbation theory to the case of nonresonant forcing and also retained the leading terms of the second order in the forcing amplitude. The result can be expressed as $\dot{R}=-\alpha_{\text {eff }} / R+V \sin \left(\Delta \omega t+\phi_{0}\right)$, where $\alpha_{\text {eff }}$ is the effective filament tension coefficient, $V$ is a coefficient, $t$ is time, $\phi_{0}$ is a constant determined by the phase of forcing, and $\Delta \omega$ is the difference $\left(\omega_{\mathrm{f}}-\omega_{0}\right)$ between the forcing frequency $\omega_{\mathrm{f}}=2 \pi / T_{\mathrm{f}}$ and the rotation frequency $\omega_{0}=2 \pi / T_{0}$ of spiral waves. The coefficient $V$ in the last term is proportional to the forcing amplitude $b_{\mathrm{f}}$. The filament tension coefficient is effectively renormalized by periodic forcing and is given by $\alpha_{\text {eff }}=\alpha+\zeta\left(b_{\mathrm{f}}^{2} / \Delta \omega\right)$, where $\zeta$ is a positive coefficient determined only by the properties of the excitable medium.

Hence, the theory predicts that rapid forcing with $\omega_{f}>\omega_{0}$ will effectively increase the filament tension and enhance collapse. If the filament tension in the medium is negative $(\alpha<0)$, it can be changed to positive tension by sufficiently strong rapid forcing, which would therefore prevent the expansion of scroll rings and lead to the shrinking of existing scroll filaments. Slow forcing with $\omega_{\mathrm{f}}<\omega_{0}$ should have the opposite effect. It lowers the filament tension and may make it negative, inducing turbulence in the medium. The last term in the evolution equation im- 


\section{R E P O R T S}

Fig. 3. The action of periodic forcing on scroll rings. (A) Time dependence of the filament length $(L)$ for an expanding scroll ring in the absence (black line) and presence (gray line) of periodic forcing. The medium parameters are the same as in Fig. 2. Periodic forcing is as described in Fig. 2 but with amplitude $b_{\mathrm{f}}=$ 0.03 and frequency $\omega_{\mathrm{f}}$ $=1.27$. (B) Time dependence of the filament length for a collapsing scroll ring in the absence (black line) and presence (gray line) of

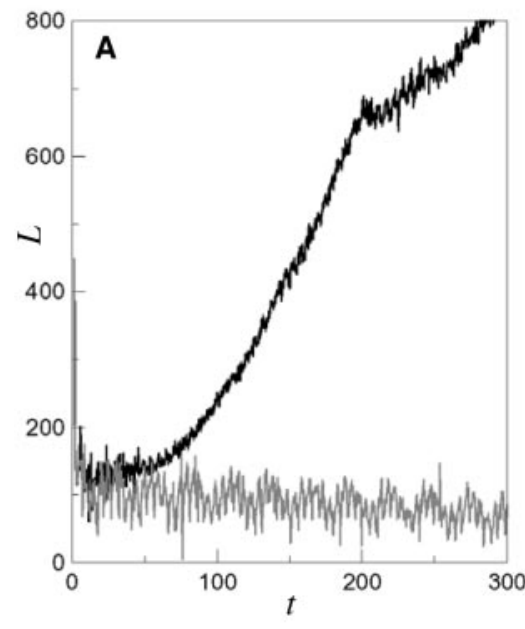

periodic forcing. The parameters are $a=1.1, b_{0}=0.16, \varepsilon=0.02, b_{\mathrm{f}}=0.025$ and $\omega_{\mathrm{f}}=0.8$; the frequency of spiral waves is $\omega_{0}=1.44$. (C) Minimal forcing amplitude $b_{f}$, needed to stabilize expanding scroll rings and suppress turbulence, as a function of the forcing frequency $\omega_{\mathrm{f}}$ for the medium with $a=1.1$, $b_{0}=0.19, \varepsilon=0.02$, and spiral wave frequency $\omega_{0}=1.19$. Black dots
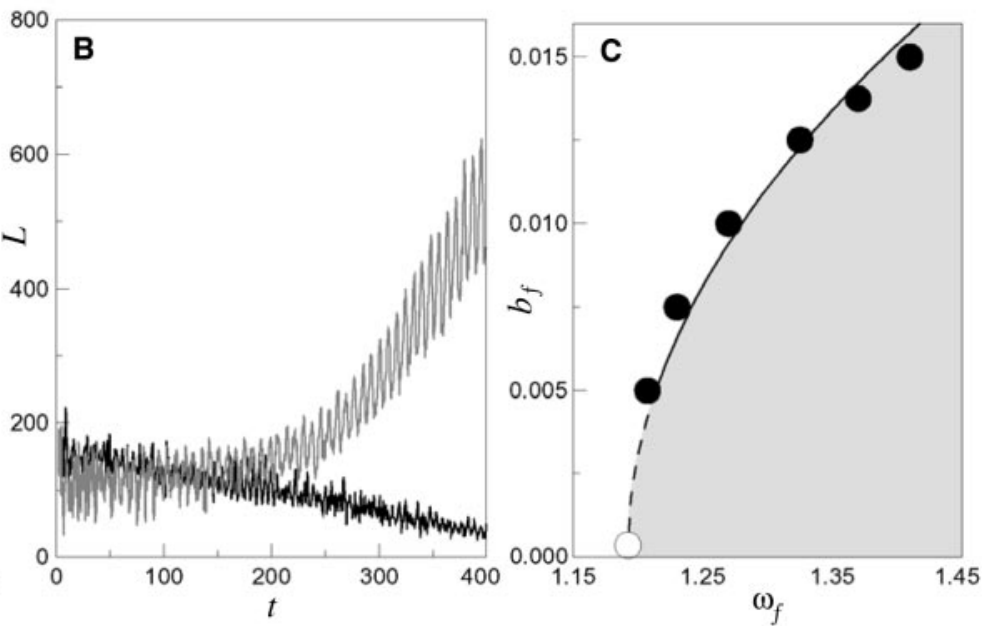

represent forcing parameters for which stabilized scroll rings have been found in simulations; the open dot indicates the rotation frequency of spiral waves $\left(\omega_{0}\right)$. The ES-CS boundary is never crossed under such weak forcing. The solid line was drawn by fitting the simulation data to the theoretical dependence $b_{\mathrm{f}}=A\left(\omega_{\mathrm{f}}-\omega_{0}\right)^{1 / 2}$ with the fitting coefficient $A=0.034$. plies that forced scroll rings should additionally breathe with period $T=2 \pi / \Delta \omega$ and amplitude inversely proportional to the frequency difference $\Delta \omega(27)$.

To test these theoretical predictions, we performed a series of simulations by varying the forcing amplitude $b_{\mathrm{f}}$ and frequency $\omega_{\mathrm{f}}$, while keeping the medium properties constant. The results have allowed us to determine the boundary that separates regions of expanding and collapsing rings in the parameter plane $\left(b_{\mathrm{f}}, \omega_{\mathrm{f}}\right)$. Along this boundary line, the effective filament tension coefficient should vanish $\left(\alpha_{\text {eff }}=0\right)$, and thus the boundary can be expressed as $b_{\mathrm{f}} \propto\left(\omega_{\mathrm{f}}-\omega_{0}\right)^{1 / 2}$. Figure $3 \mathrm{C}$ shows that this relationship was indeed satisfied in numerical simulations. On the boundary, breathing scroll rings, which on the average neither collapse nor expand, were observed under periodic forcing.

Efficient control of scroll waves and turbulence was achieved only by nonresonant periodic forcing. If the difference $\Delta \omega$ between the forcing frequency and the rotation frequency of spiral waves in the medium was small, the breathing of scroll waves was strongly enhanced and became slow. When almost-resonant forcing was applied, largemagnitude irregular oscillations of filaments were found in the simulations and stabilization of the scroll rings was impossible.

In addition to negative filament tension, scroll waves in excitable media have other instabilities $(7,28)$. However, these instabilities develop at sufficiently high excitability, when interaction between waves becomes significant. In our study, we avoided region MS of the parameter space (Fig. 1), where meandering of spiral waves is found in two dimensions. Moreover, spontaneous breakup of spiral waves does not take place in the Barkley model. We investigated scroll wave patterns and turbulence under conditions where the medium completely recovered after each excitation.

Although the numerical analysis was performed here only for a particular model, its results may be typical for a broad class of excitable media. Indeed, the observed behavior was also found in the kinematic description, where an excitable medium is specified by only a few general phenomenological parameters. In the system considered here, diffusive spreading of the inhibitor was absent, a condition characteristic of cardiac tissue and of the BelousovZhabotinsky reaction in gels with an immobilized catalyst. Simulations with a modified model, including inhibitor diffusion, indicate that the parameter region occupied by expanding scroll rings diminishes and disappears when diffusion constants of the activator and the inhibitor become equal (26). Thus, the phenomenon of Winfree turbulence should be more difficult to observe in aqueous chemical solutions. Generally, negative tension of vortex filaments is expected at relatively low excitability.

We introduced periodic forcing of wave patterns through periodic uniform variation of the excitation threshold, but similar effects are apparently also possible when other properties of an excitable medium are periodically modulated. As we have demonstrated, the action of forcing on Winfree turbulence markedly depends on the relationship between the period of forcing and the rotation period of the spiral waves. In this model, the period of spiral waves could easily be found by running independent two-dimensional (2D) simulations. However, we have also determined the Fourier spectrum of Winfree turbulence and have seen that it always possessed a maximum at a frequency close to the rotation frequency of spiral waves. To suppress turbulence, sufficiently strong rapid forcing at a frequency above this spectral maximum should be applied.

Control of Winfree turbulence may have important applications in cardiology as a new approach for terminating certain types of ventricular fibrillation. Further numerical and analytical studies are needed to systematically investigate the taming of Winfree turbulence in a broad-parameter region and in realistic models of cardiac tissue.

References and Notes

1. A. T. Winfree, Science 266, 1003 (1994).

2. R. A. Gray et al., Science 270, 1222 (1995)

3. A. V. Panfilov, P. Hogeweg, Science 270, 1223 (1995).

4. A. V. Panfilov, A. N. Rudenko, Phys. D 28, 215 (1987).

5. P. K. Brazhnik, V. A. Davydov, V. S. Zykov, A. S. Mikhailov, Sov. Phys. JETP 66, 984 (1987).

6. V. N. Biktashev, A. V. Holden, H. Zhang, Philos. Trans. R. Soc. London Ser. A 347, 611 (1994).

7. H. Henry, V. Hakim, Phys. Rev. Lett. 85, 5328 (2000)

8. F. H. Fenton, E. M. Cherry, H. M. Hastings, S. J. Evans, Chaos 12, 852 (2002).

9. A. T. Winfree, Science 181, 937 (1973).

10. F. Siegert, C. J. Weijer, Proc. Natl. Acad. Sci. U.S.A. 89, 6433 (1992).

11. V. Petrov, Q. Ouyang, H. L. Swinney, Nature 388, 655 (1997).

12. O. U. Kheowan, V. S. Zykov, O. Rangsiman, S. C. Müller, Phys. Rev. Lett. 86, 2170 (2001).

13. T. Sakurai, E. Mihaliuk, F. Chirilla, K. Showalter, Science 296, 2009 (2002).

14. A. L. Lin et al., Phys. Rev. Lett. 84, 4240 (2000)

15. V. K. Vanag, L. Yang, M. Dolnik, A. M. Zhabotinsky, I. R. Epstein, Nature 406, 389 (2000)

16. M. Kim et al., Science 292, 1357 (2001).

17. M. Vinson, S. Mironov, S. Mulvey, A. M. Pertsov, Nature 386, 477 (1997).

18. T. Ameniya, P. Kettunen, S. Kádár, T. Yamaguchi, K. Showalter, Chaos 8, 872 (1998).

19. R. M. Mantel, D. Barkley, Phys. D 149, 107 (2001).

20. V. Pérez-Muñuzuri, F. Sagués, J. M. Sancho, Phys. Rev. E 62, 94 (2000).

21. D. Barkley, M. Kenss, L. S. Tuckerman, Phys. Rev. A 42, 2489 (1990). 
22. B. Fiedler, R. M. Mantel, Doc. Math. 5, 695 (2000) 23. J. P. Keener, J. J. Tyson, Science 239, 1284 (1988).

24. A. S. Mikhailov, Foundations of Synergetics I. Distributed Active Systems (Springer, Berlin, ed. 2, 1995).

25. A. Yu. Abramychev, V. A. Davydov, A. S. Mikhailov, Biophysics 35, 520 (1990).

26. S. Alonso, F. Sagués, A. S. Mikhailov, in preparation.

27. The higher perturbation terms, responsible for renormalization of the filament tension coefficient, have been computed under an assumption that the amplitude of breathing of a ring filament is small as compared with its radius. Because the amplitude of breathing diverges in the limit $\Delta \omega \rightarrow 0$, this imposes a restriction near the resonance at $\Delta \omega=0$.

28. I. Aranson, I. Mitkov, Phys. Rev. E 58, 4556 (1998).

29. The authors acknowledge financial support from the REACTOR program of the European Science Foundation and from Dirección General de Investigación
(Spain) under project BXX2000-0638-C02. S.A. was financially supported by a grant from the Ministerio de Ciencia y Tecnología (Spain).

6 November 2002; accepted 16 January 2003 Published online 30 January 2003;

$10.1126 /$ science. 1080207

Include this information when citing this paper.

\section{Recent Trends in Arctic Surface, Cloud, and Radiation Properties from Space}

\author{
Xuanji Wang ${ }^{1 *}$ and Jeffrey R. Key ${ }^{2}$
}

Trends in satellite-derived cloud and surface properties for 1982 to 1999 show that the Arctic has warmed and become cloudier in spring and summer but has cooled and become less cloudy in winter. The increase in spring cloud amount radiatively balances changes in surface temperature and albedo, but during summer, fall, and winter, cloud forcing has tended toward increased cooling. This implies that, if seasonal cloud amounts were not changing, surface warming would be even greater than that observed. Strong correlations with the Arctic Oscillation indicate that the rise in surface temperature and changes in cloud amount are related to large-scale circulation rather than to local processes.

Recent studies have shown that Northern Hemisphere sea-ice extent and thickness have been decreasing $(1,2)$, whereas land surface air temperature has increased markedly over the past 30 years (3). Arctic climate change has also been noted in the horizontal flux of precipitable water (PW), snowfall, and vegetation (4-6). Although these studies generally agree that the Arctic has been warming, it is not clear how other aspects of the climate system have responded. Have cloud characteristics changed? How do changes in surface and cloud properties interact with and affect the surface radiation budget; i.e., what is the cloud-radiation feedback?

Data from the Advanced Very High Resolution Radiometer (AVHRR) Polar Pathfinder (APP) project (7) for the period 1982 to 1999 are used to estimate surface, cloud, and radiation properties. The daily data are centered on a local solar time of 14:00 (high sun) and subsampled to $25-\mathrm{km}$ pixels. We have extended the standard APP product (hereafter APP-x) to include all-sky surface temperature and broadband albedo, cloud properties (amount, particle phase, effective radius, optical depth, temperature, and pressure), radiative fluxes, and the cloud radiative effect, or cloud "forcing." Processing was done as described in (8), with atmospheric profile data from the National

${ }^{1}$ Cooperative Institute for Meteorological Satellite Studies, University of Wisconsin-Madison, 1225 West Dayton Street, Madison, WI 53706, USA. '2Office of Research and Applications, National Oceanic and Atmospheric Administration, National Environmental Satellite, Data, and Information Service, 1225 West Dayton Street, Madison, WI 53706, USA.

*To whom correspondence should be addressed. Email: xuanjiw@ssec.wisc.edu
Center for Environmental Prediction-National Center for Atmospheric Research (NCEP/ NCAR) reanalysis (9) and ozone from the
International Satellite Cloud Climatology Project (ISCCP) D2 data set (10). The extended products have been validated with data collected during Arctic field experiments and with data from Arctic and Antarctic meteorological stations (11-14).

Trend analysis of cloud, surface, and radiation parameters was performed with linear least-squares fit regression. Unless noted otherwise, all trends reported here are significant at the $90 \%$ confidence level or higher. Figure 1 shows seasonal and annual trends of surface temperature and surface albedo data over land and ocean north of $60^{\circ} \mathrm{N}$. The surface temperature has decreased at the decadal rate of $-0.34^{\circ} \mathrm{C}$ in winter (December through February) but at a confidence level of only $69 \%$. For the area north of $80^{\circ} \mathrm{N}$ (not shown), the APP-x surface temperature has decreased at $-2.2{ }^{\circ} \mathrm{C}$ per decade. The wintertime sur-

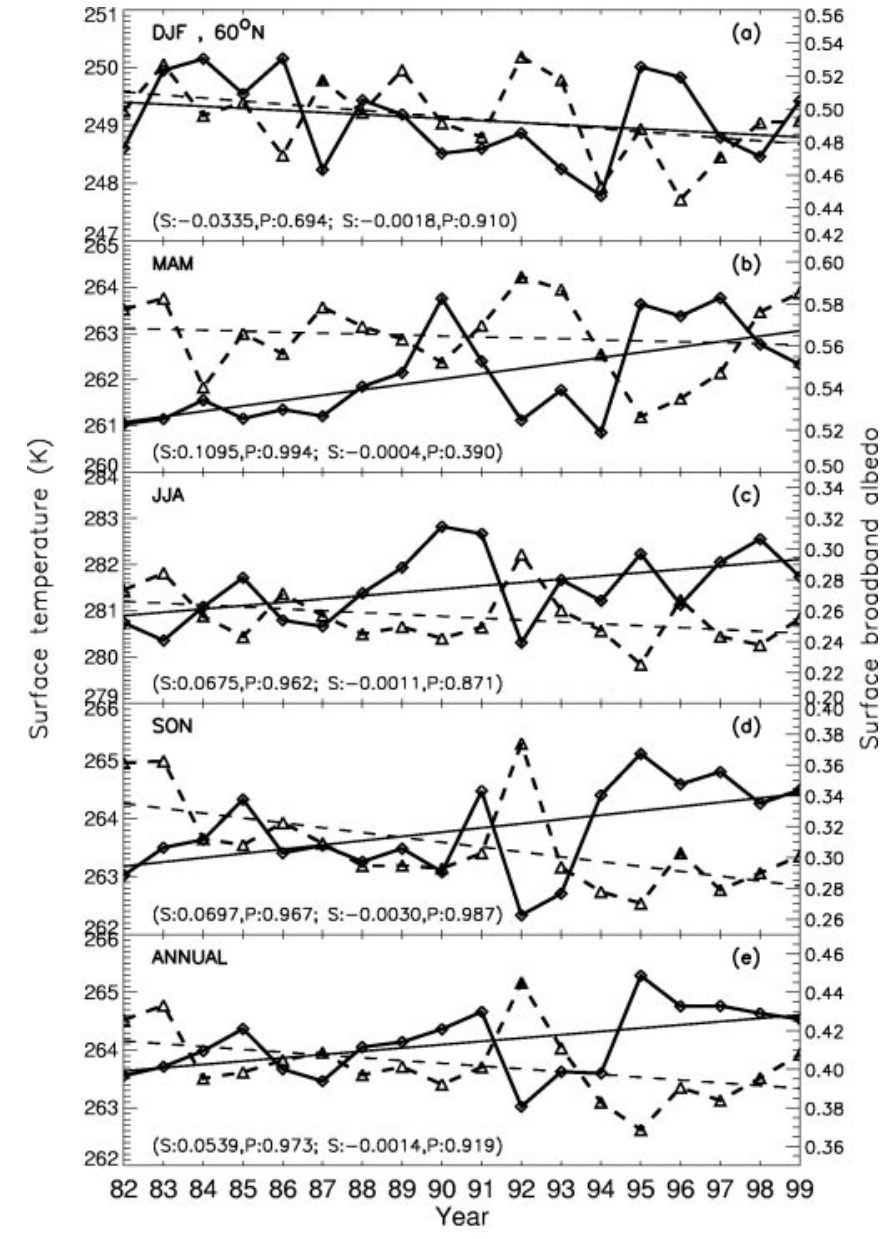

Fig. 1. Time series and trends of surface skin temperature and broadband albedo in winter [December, January, February (DJF)], spring [March, April, May (MAM)], summer [June, July, August (JJA)], and autumn [September, October, November $(\mathrm{SON})]$, and the annual mean during the period 1982 to 1999 for the Arctic north of $60^{\circ} \mathrm{N}$. The numbers in parentheses are the trend slope per year $(S)$ and the $F$ test confidence level (P). The first pair of $S$ and $P$ values denotes the surface temperature trend (solid line), and the second pair is for the surface albedo (dashed line). 